\title{
Thin Layer Chromatography Coupled with Surface-Enhanced Raman Scattering as a Facile Method for On-Site Quantitative Monitoring of Chemical Reactions
}

\author{
Zong-Mian Zhang, Jing-Fu Liu,* Rui Liu, Jie-Fang Sun, and Guo-Hua Wei \\ State Key Laboratory of Environmental Chemistry and Ecotoxicology, Research Center for Eco-Environmental Sciences, Chinese \\ Academy of Sciences, Beijing 100085, China
}

\section{Supporting Information}

ABSTRACT: By coupling surface-enhanced Raman spectroscopy (SERS) with thin layer chromatography (TLC), a facile and powerful method was developed for on-site monitoring the process of chemical reactions. Samples were preseparated on a TLC plate following a common TLC procedure, and then determined by SERS after fabricating a large-area, uniform SERS substrate on the TLC plate by spraying gold nanoparticles (AuNPs). Reproducible and strong SERS signals were obtained with substrates prepared by spraying 42-nm AuNPs at a density of $5.54 \times 10^{10} \mathrm{~N} / \mathrm{cm}^{2}$ on the TLC plate. The capacity of this TLC-SERS method was evaluated by monitoring a typical Suzuki coupling reaction of phenyl-

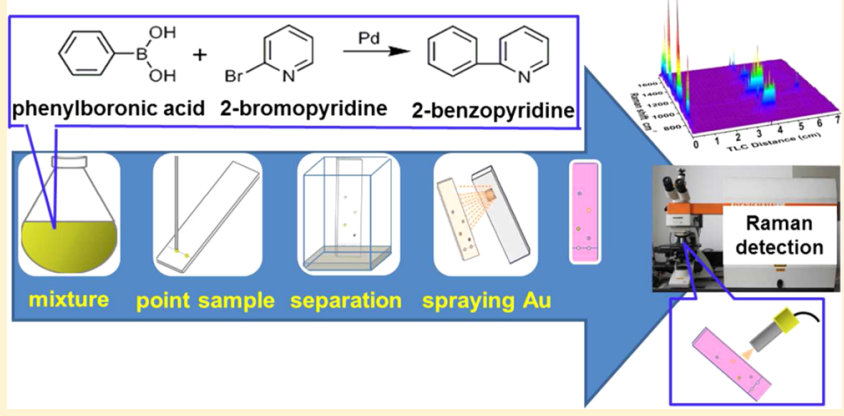
boronic acid and 2-bromopyridine as a model. Results showed that this proposed method is able to identify reaction product that is invisible to the naked eye, and distinguish the reactant 2-bromopyridine and product 2-phenylpyridine, which showed almost the same retention factors $\left(R_{\mathrm{f}}\right)$. Under the optimized conditions, the peak area of the characteristic Raman band $\left(755 \mathrm{~cm}^{-1}\right)$ of the product 2-phenylpyridine showed a good linear correlation with concentration in the range of $2-200 \mathrm{mg} / \mathrm{L}\left(R^{2}=0.9741\right)$, the estimated detection limit $(1 \mathrm{mg} / \mathrm{L} 2$-phenylpyridine) is much lower than the concentration of the chemicals in the common organic synthesis reaction system, and the product yield determined by the proposed TLC-SERS method agreed very well with that by UPLC-MS/MS. In addition, a new byproduct in the reaction system was found and identified through continuous Raman detection from the point of sample to the solvent front. This facile TLC-SERS method is quick, easy to handle, low-cost, sensitive, and can be exploited in on-site monitoring the processes of chemical reactions, as well as environmental and biological processes.

$\mathrm{F}$ or their advantages like simple operation and fast - separation, thin layer chromatography (TLC)-based separation technologies endow a lasting popularity in various research fields, and they play a major role in monitoring synthetic organic reactions. ${ }^{1,2}$ Generally, after spotted on the plate and developed in the mobile phase via capillary action, the separated sample spots are visualized by the absorbance or fluorescence, either native or enhanced with visualizing agents, and the relevant products are identified by their characteristic colors or fluorescence combined with the retention factor $\left(R_{\mathrm{f}}\right)$ values. However, the following challenges remain:

(i) because of the limited specificity and sensitivity provision capacity of TLC, spectroscopic routines like nuclear magnetic resonance (NMR) and mass spectroscopy (MS) are needed to further characterize reaction products after the extraction of the chemicals from the TLC plate, ${ }^{3,4}$ which is time-consuming and laborintensive;

(ii) some chemicals like alkanes are fluorescence-insensitive, and cannot be detected under an ultraviolet (UV) lamp, thus prohibiting their monitoring; and (iii) since the separation on TLC is mainly based on the chemicals' different affinity with the stationary and mobile phase, chemicals with similar structure or polarity are difficult to separate completely.

To overcome these limits, various technologies, such as infrared (IR), Raman, and mass spectrometry have been explored with TLC for compound specific information. Among these technologies, special attention has been paid to surfaceenhanced Raman scattering (SERS), partly for its highsensitivity with detection limit down to the single-molecule level under optimized conditions, ${ }^{5}$ fingerprint-based high specificity and capacity in simultaneous detection of multianalytes, ${ }^{6}$ and high tolerance to various analyte configurations including liquid, powder, gas and solid samples like foods ${ }^{7,8}$ and artworks. ' Furthermore, the development in instrument automation enables the fulfillment of large area mapping with

Received: May 9, 2014

Accepted: June 30, 2014

Published: June 30, 2014 
Scheme 1. Schematic Representation of the TLC-SERS Study of Continuous Raman Scanning

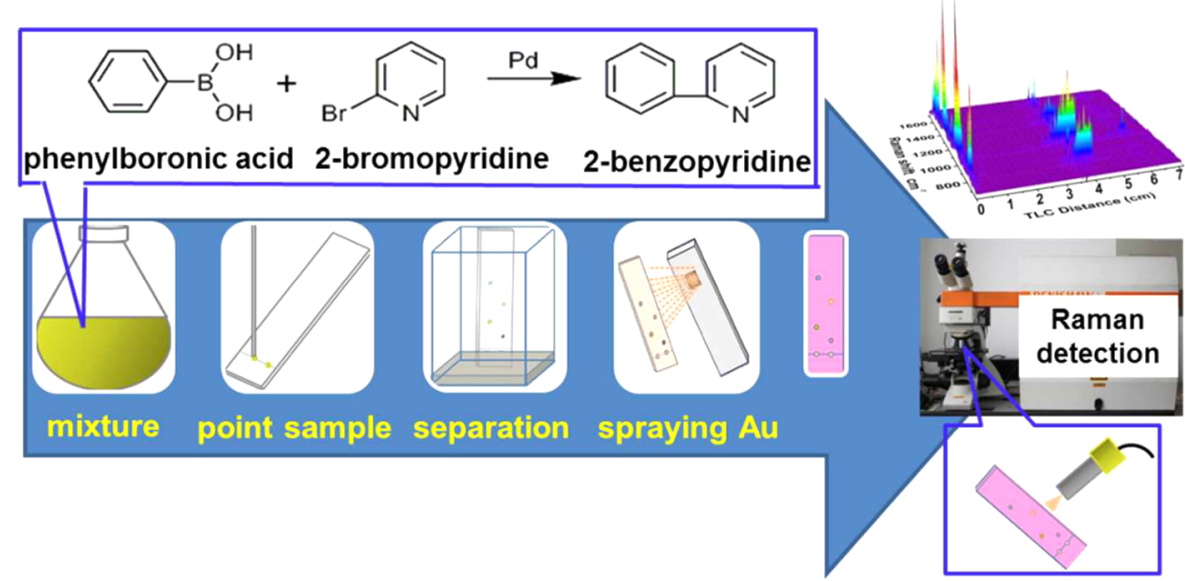

Raman spectrometer in subminute time scale. Therefore, the combination of SERS and TLC enables the conduction of separation and detection on the same plate, and distinguishment of the incompletely separated compounds from their characteristic Raman bands, without loss of chemicals through the imaging-mode of SERS.

Since first reported by Hezel, ${ }^{10}$ TLC-SERS had been applied to the analysis of various analytes, ${ }^{11-13}$ including the natural dyes on artwork, ${ }^{9}$ pesticide residue from crops, ${ }^{14}$ some biomarkers in biological samples, ${ }^{15-18}$ and on-site detection of aromatic pollutants in environmental water samples. ${ }^{19}$ In most of the above methods, silver nanoparticles (AgNPs) was dropped on the spots of preseparated TLC plate, and then the SERS spectrum was collected from each spot for the information on the surface analytes. Therefore, these methods are only valid for the analysis of visible analytes on the plate in the millimeter-sized area $\left(\mathrm{mm}^{2}\right)$, but not valid for automatic and continuous analysis of all the chemicals, including invisible analytes on the plate. Freye et al. ${ }^{20}$ realized the analysis in larger area and distinguished the compounds with similar $R_{\mathrm{f}}$ values through establishing a conformal blotting process and transferring analytes from TLC plate to nanocomposite films. However, the preparation of silver-polymer nanocomposites and a transferring procedure are required, which is very complicated and time-consuming.

Herein, we report a new TLC-SERS method for continuous and automatic on-site monitoring of chemical reaction processes on the TLC plate. After separation of the products on a commercially available TLC plate, SERS substrate with high uniformity of gold nanoparticles (AuNPs) was fabricated on the plate by spraying Au colloid on it, and then the SERS scanning was conducted along a line from the point of sample to the solvent front at submicrometer-scale intervals. The SERS spectra along the line provide all the information on the separated components, including fingerprint spectra for identification of chemicals that are incompletely separated or invisible to the eyes on the plate.

\section{EXPERIMENTAL SECTION}

Chemicals and Materials. Chlorauric acid $\left(\mathrm{HAuCl}_{4}\right)$, sodium citrate, potassium phosphate, petroleum ether, and ethyl acetate were purchased from Sinopharm Chemical Reagent Co. (Beijing, China). Palladium acetate, palladium nitrate, and palladium chloride were supplied by Aladdin
Reagent Database, Inc. (Shanghai, China). Phenylboronic acid, 2-bromopyridine, 2-phenylpyridine, biphenyl, and 2,2-bipyridine were purchased from Sigma-Aldrich (St. Louis, MO). Silica gel TLC plates ( $\mathrm{G}$ and $\mathrm{GF}_{254}$ ) were acquired from Anhui LiangChen Silicon Material Co. (Anhui, China). The sprayer was purchased from Ningbo Danlong Electric Manufacturer Co. (Zhejiang, China). All the chemicals are at least analytic grade, and pure water produced with a Millipore Milli-Q Gradient system (Billerica, MA) was used throughout this study.

Organic Synthesis Procedure. The Suzuki coupling reaction of phenylboronic acid and 2-bromopyridine was chosen as the model for evaluating the developed TLC-SERS method. The synthesis reaction was carried out in a threenecked bottle under constant stirring. The $10 \mathrm{~mL}$ of ethanol/ water (1:1) solvent containing $2.5 \mathrm{mmol}$ potassium phosphate was poured into the three-necked bottle, followed by adding 1.3 mmol 2-bromopyridine, $1.9 \mathrm{mmol}$ phenylboronic acid, and 17.8 $\mu$ mol palladium acetate. The reaction solution was performed at room temperature to slow the reaction rate, which can fulfill the requirement of the monitoring.

Synthesize and Characterization of AuNPs. AuNPs were prepared using sodium citrate as the reducing and capping agent according to the literature. ${ }^{21}$ Briefly, $150 \mathrm{~mL}$ of $1 \mathrm{mmol} /$ $\mathrm{L}$ chlorauric acid solutions was heated to boil. After adding appropriate amounts of sodium citrate and continued boiling and stirring of the mixture for $\sim 30 \mathrm{~min}$, different sizes of AuNPs were obtained in the solutions. The produced AuNPs were characterized by transmission electron microscopy (TEM), and results are shown in Figure S1 in the Supporting Information (SI).

TLC Separation. After being activated by heating at $110^{\circ} \mathrm{C}$ for $30 \mathrm{~min}$, the silica gel TLC plate was micropipetted with 1$\mu \mathrm{L}$ samples. After drying in the air, the plate was placed in a $125-\mathrm{mL}$ chamber and developed using petroleum ether and ethyl acetate $(\mathrm{v} / \mathrm{v}=5: 1)$ as the mobile phase solvent. After the separation of the samples, Au dispersions were sprayed onto the plate with a sprayer. The sprayer allowed aqueous AuNP dispersions to form fine drops of colloid (in nanometer range) to uniformly coat the TLC plate. In this way, the plate surface was entirely covered with uniform AuNPs without diffusion of the chemical spots. The size and amount of AuNPs sprayed on the TLC plate was optimized to obtain the best SERS signal. 


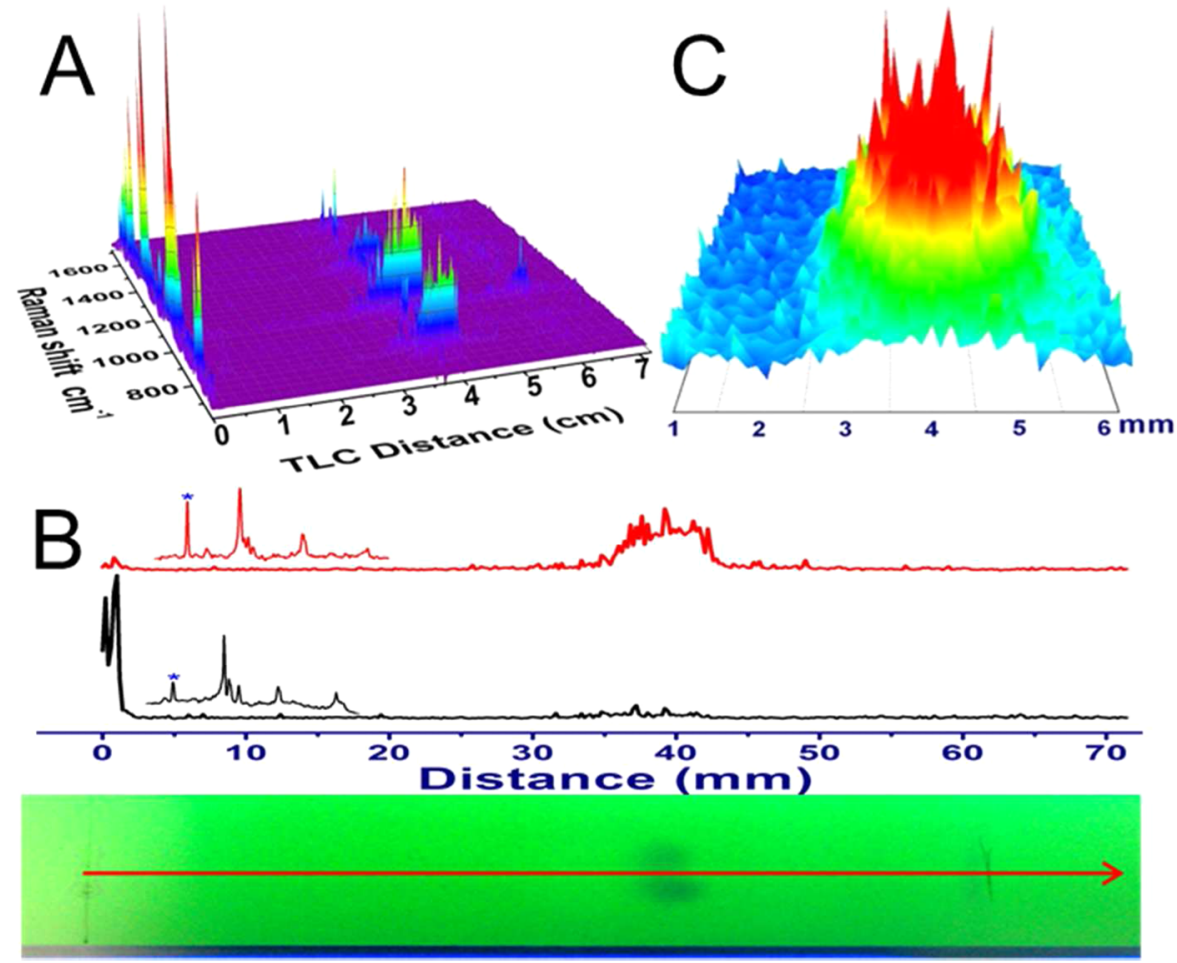

Figure 1. (A) Raman image from the point of the sample to the solvent front along a line; (B) comparison of Raman signal distribution of 2phenylpyridine (red, $755 \mathrm{~cm}^{-1}$ ) and phenylboronic acid (black, $737 \mathrm{~cm}^{-1}$ ) on the plate with the fluorescence image of the TLC plate; (C) Raman image of 2-phenylpyridine in the product area.

SERS Detection. For the Raman spectra collection, a Renishaw Invia Raman microscope equipped with a 785-nm laser was used, and a $20 \times$ long working-distance objective was used with an output power of $1 \mathrm{~mW}$ and a $20-\mu \mathrm{m}$ beam diameter on the plate. The TLC plate was placed on the XYZ stage of the Raman spectrometer and scanned from the sample point to the solvent front at $300 \mu \mathrm{m}$ interval along a line, and all the spectra were scanned from $618 \mathrm{~cm}^{-1}$ to $1719 \mathrm{~cm}^{-1}$. The SERS signals were obtained and mapped with Raman shift as the $X$-axis, movement distance as the $Y$-axis, and the Raman signal intensity as the $Z$-axis. In this way, the SERS spectra of all the chemicals along the line were recorded in several minutes and the concentration of the chemicals were obtained at the same time. The detection can be finished in several minutes (Scheme 1).

UPLC-MS/MS Determination of Product. For ultraperformance liquid chromatography (UPLC) monitoring the reaction, $10 \mu \mathrm{L}$ of reaction solution was sampled at different reaction time and terminated by adding $40 \mu \mathrm{L}$ of $0.4 \mathrm{~mol} / \mathrm{L}$ acetic acid solutions, and then diluting to suitable concentrations with the $10 \mathrm{mmol} / \mathrm{L}$ acetic acid. The reaction product (2-phenylpyridine) was determined by a TSQ Quantum Access triple quadruple mass spectrometer (Thermo Scientific, Waltham, MA) coupled with a Waters Acquity UPLC (Waters, Milford, MA). Separation of the products was accomplished using the UPLC system equipped with a Waters $\mathrm{BEH} \mathrm{C}_{18}$ column $(2.1 \mathrm{~mm} \times 50 \mathrm{~mm}, 1.7 \mu \mathrm{m})$. The injection volume was $10 \mu \mathrm{L}$, and the column temperature was $45{ }^{\circ} \mathrm{C}$. The mobile phase was a mixture of $0.1 \%$ formic acid and methanol $(\mathrm{v} / \mathrm{v}=$ 1:1) with a flow rate of $0.2 \mathrm{~mL} / \mathrm{min}$. The retention time of the product was $1.37 \mathrm{~min}$. For the mass spectrometer detection, the electrospray ionization (ESI) was operated in the positive-ion mode, and the best conditions were set as follows: spray voltage
$=4000 \mathrm{~V}$ and capillary temperature $=300{ }^{\circ} \mathrm{C}$. Nitrogen was used as the sheath gas (35 units) and auxillary gas (20 units).

\section{RESULTS AND DISCUSSION}

Method Development and Analytical Performance. Figure 1A shows the SERS spectra of reaction solution of the Suzuki coupling of phenylboronic acid and 2-bromopyridine. To avoid the interference from each other, the Raman bands of 737 and $755 \mathrm{~cm}^{-1}$ were used for the quantification of phenylboronic acid and 2-phenylpyridine, respectively, and the distribution of the two chemicals on the plate was found to match well with the spots on the $\mathrm{GF}_{254}$ TLC plate (Figure 1B). To test the applicability of this method for detection in larger areas, the spot of the 2-phenylpyridine $(6 \mathrm{~mm}$ in diameter, visualized under a 254-nm UV lamp) was detected in a spectral acquisition density of $0.1 \mathrm{~mm} \times 0.1 \mathrm{~mm}$. The over 3000 SERS spectra obtained were mapped based on the characteristic Raman band of $755 \mathrm{~cm}^{-1}$, which corresponds to the totally symmetric in-plane vibration of phenyl ring. ${ }^{22,23}$ As shown in Figure 1C, the Raman signal intensity of 2-phenylpyridine distributed like a torch light in the measuring area, which represents its concentration distribution. The above results suggested that this method can be used for monitoring of a process by continuous detection in large area, and the missing of analytes can be avoided by using the image mode at the interval of $300 \mu \mathrm{m}$.

The size and concentration of AuNPs for fabricating the SERS substrates were optimized to improve the method sensitivity. Environmental scanning electron microscopy (ESEM) surface images showed the presence of a difference in structure of the SERS substrates fabricated with different sizes of AuNPs $(18,33,42,71 \mathrm{~nm})$ on the TLC plate (Figure S2 in the Supporting Information). The SERS spectra of series 

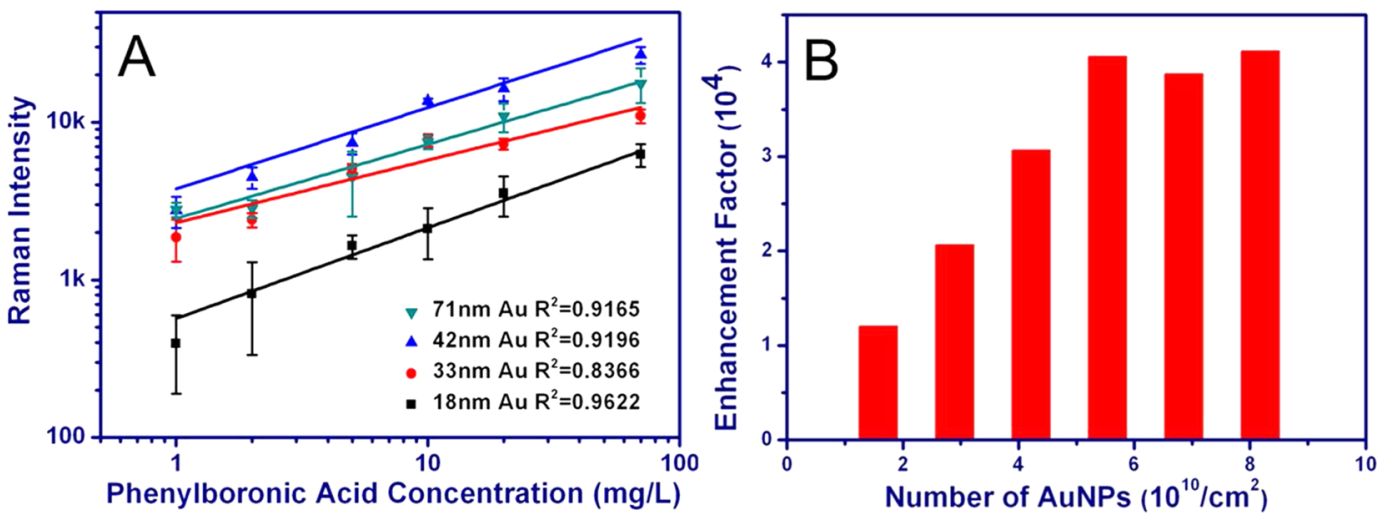

Figure 2. Impacts of AuNP size and concentration on the SERS response. (A) SERS intensity detected with SERS substrates fabricated from different-sized AuNPs; the concentrations of phenylboronic acid were 1, 2, 5, 10, 20, and $70 \mathrm{mg} / \mathrm{L}$. (B) The Raman-enhanced factor for phenylboronic acid detected by spraying with $1.58 \times 10^{10}, 2.9 \times 10^{10}, 4.22 \times 10^{10}, 5.54 \times 10^{10}, 6.86 \times 10^{10}$, and $8.18 \times 10^{10} \mathrm{~N} / \mathrm{cm}^{2} \mathrm{AuNPs}(42 \mathrm{~nm})$ on the TLC plate. A Raman spectrometer was used with a laser intensity of $1 \mathrm{~mW}$, an acquisition time of $1 \mathrm{~s}$, and two accumulate times.
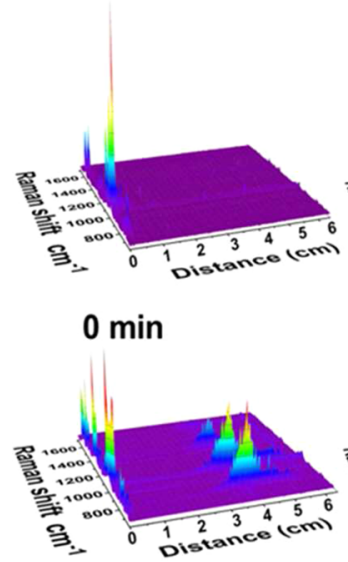

$140 \mathrm{~min}$
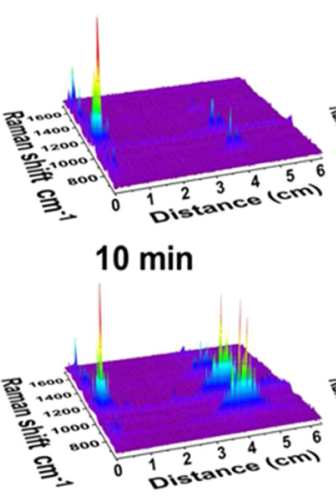

$50 \mathrm{~min}$

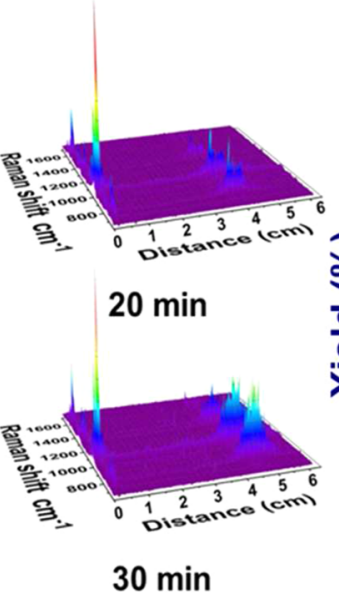

$30 \mathrm{~min}$

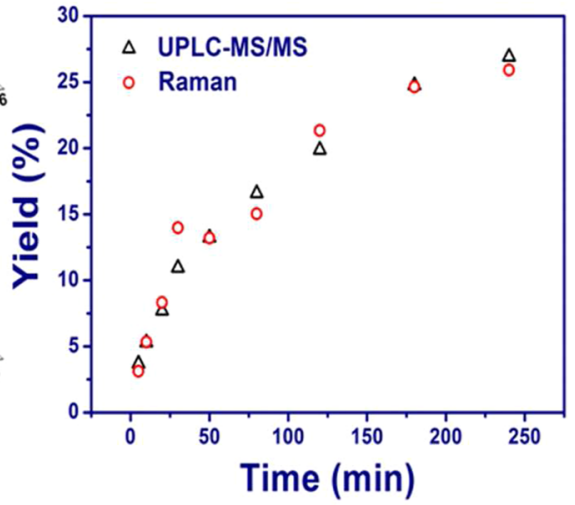

Figure 3. (Left) Monitoring the Suzuki coupling reaction of phenylboronic acid and 2-bromopyridine at different reaction times. (Right) Product yield determined by Raman and UPLC-MS/MS at different reaction times.

concentrations of phenylboronic acid (1-70 mg/L) were detected with SERS substrates formed by spraying differentsized AuNPs on the preseparated TLC plate pointed with phenylboronic acid samples. Considering the roughness of the TLC plate surface, 11 spectra of each sample spot were collected and the average value was adopted to calculate the SERS signal intensity. Figure 2A shows the SERS intensity of $999 \mathrm{~cm}^{-1}$ Raman band, the strongest breathing mode $\left(\nu_{12}\right)$ of phenyl ring, ${ }^{24}$ at a series of phenylboronic acid concentrations, indicating that 42-nm AuNPs showed the best performance.

The concentration of 42-nm AuNPs for fabricating the SERS substrates was optimized by spraying different concentrations of AuNPs onto the preseparated TLC plates pointed with 10 $\mathrm{mg} / \mathrm{L}$ phenylboronic acid as the probe, and calculating the enhanced factors (EFs) by comparing the Raman intensity at the $999 \mathrm{~cm}^{-1}$ band to the bulk Raman signal obtained from the TLC plate with $2 \mathrm{~g} / \mathrm{L}$ phenylboronic acid. The AuNP concentration was calculated based on the equation given by eq $1:^{25,26}$

$$
C_{\text {AuNPs }}=\frac{C_{\text {chlorauric acid }}}{4 \pi(R-\delta)^{3} / 3 V_{g}}
$$

where $C_{\text {chlorauric acid }}$ is the concentration of $\mathrm{HAuCl}_{4}, R$ the radius of AuNPs, $\delta$ the length of the protecting ligand $(\delta \ll R)$, and $V_{\mathrm{g}}$ the volume of one $\mathrm{Au}$ atom $\left(17 \AA^{3}\right)$. The EF was calculated according to eq 2 :

$$
R_{\mathrm{EF}}=\frac{I_{\mathrm{SERS}}}{I_{\text {bulk }}}\left(\frac{N_{\text {bulk }}}{N_{\text {SERS }}}\right)\left(\frac{P_{\text {bulk }}}{P_{\text {SERS }}}\right)\left(\frac{t_{\text {bulk }}}{t_{\text {SERS }}}\right)
$$

in which $I$ represents the Raman intensity, $N$ is the number of probe molecules, $P$ is the laser power, and $t$ represents the acquisition time; and subscripts "SERS" and "bulk" represent surface-enhanced Raman spectroscopy and normal Raman scattering of bulk solution, respectively. Results shown in Figure $2 \mathrm{~B}$ indicated that the EFs increased with AuNP concentration until reaching a value of $5.5 \times 10^{10} \mathrm{~N} / \mathrm{cm}^{2}$ and then leveled off, suggesting that AuNPs at this concentration on the TLC plate provided suitable spacing between the particles and the target molecules. In the following experiments, 42-nm AuNPs were sprayed on the TLC plate to provide concentration of $5.5 \times 10^{10} \mathrm{~N} / \mathrm{cm}^{2}$.

Under the above optimized conditions, the SERS images of 2-phenylpyridine $\left(755 \mathrm{~cm}^{-1}\right)$ at various concentrations were recorded, and the peak area of the characteristic Raman band $\left(755 \mathrm{~cm}^{-1}\right)$ showed a good linear correlation with 2- 
phenylpyridine concentrations in the range of $2-200 \mathrm{mg} / \mathrm{L}\left(R^{2}\right.$ $=0.9741$ ) (see Figure S3 in the Supporting Information). The estimated detection limit is $1 \mathrm{mg} / \mathrm{L} 2$-phenylpyridine, which is much lower than the concentration of the chemicals in the common organic synthesis reaction system, verifying the great applicability of this method in on-site monitoring of organic synthesis process.

Application in Monitoring the Reaction Process. The proposed TLC-SERS method was employed to monitor the Suzuki reaction process through the SERS detection of phenylboronic acid and 2-bromopyridine using the $\mathrm{GF}_{254}$ TLC plate as the separation and detection substrate. Figure 3 (left) shows the SERS images of the preseparated mixture recorded at different reaction times. The Raman signal intensity of the product (2-phenylpyridine) increased constantly with time up to $50 \mathrm{~min}$ and then remained constant, suggesting that either the reaction has reached equilibrium or the Raman signal intensity of the product had reached saturation under the given instrument conditions. To further verify the reason, the reaction solution can be diluted and analyzed again under the same conditions.

The applicability of the proposed method was further verified by monitoring the yields of the product, with UPLC-MS/MS detection as a reference method. Results (Figure 3 (right)) showed that the product yield increased quickly with the time up to $200 \mathrm{~min}$ and then continued to experience slow growth to the end. The profiles of product yield to reaction time obtained by these two methods agreed very well, indicating the reliability of TLC-SERS in quantification of the analytes. Given that the TLC-SERS method is much simpler and quicker than the UPLC-MS/MS approach, the TLC-SERS is a promising alternative for monitoring the process, both qualitatively and quantitatively.

Isolation and Identification of Analytes in Mixture. It is interesting to note that after the development of the Suzuki coupling reaction mixture of phenylboronic acid and 2bromopyridine on the $\mathrm{GF}_{254}$ TLC plate, no Raman signal of 2-bromopyridine was detected, even at the initial stage of the reaction. We ascribed it to the swamping of the 2bromopyridine signals in the green fluorescence background of the $\mathrm{GF}_{254}$ TLC plate. This speculation was verified by the distinct Raman signal of 2-bromopyridine detected by using the G TLC plate under the same detection conditions as that with $\mathrm{GF}_{254}$ TLC plate (see Figure S4 in the Supporting Information).

When G TLC plate was used, however, the Raman intensity of the product was found to remain almost constant during the reaction process (see Figure S5 in the Supporting Information). We conjectured that 2-bromopyridine and 2-phenylpyridine have similar $R_{\mathrm{f}}$ values and overlapped Raman spectra after TLC separation, thus the product signal balanced due to the increase of product 2-phenylpyridine and the decrease of reactant 2bromopyridine with the reaction time. To verify this speculation, 2-bromopyridine, 2-phenylpyridine, and their mixture were respectively pointed on the same G TLC plate, while 2-bromopyridine and 2-phenylpyridine were respectively pointed on the same $\mathrm{GF}_{254}$ TLC plate. After development under the same conditions, nothing can be seen on the G TLC plate under the 254-nm UV light (Figure 4A). However, with the characteristic Raman band of $703 \mathrm{~cm}^{-1}\left(\nu_{\mathrm{C}-\mathrm{Br}}\right)$ for 2bromopyridine $^{27}$ and $755 \mathrm{~cm}^{-1}\left(\nu_{\mathrm{Ph}}\right)$ for 2-phenylpyridine, Raman mapping can be made continuously on the G TLC plate from the point of sample to the solvent front. Results showed

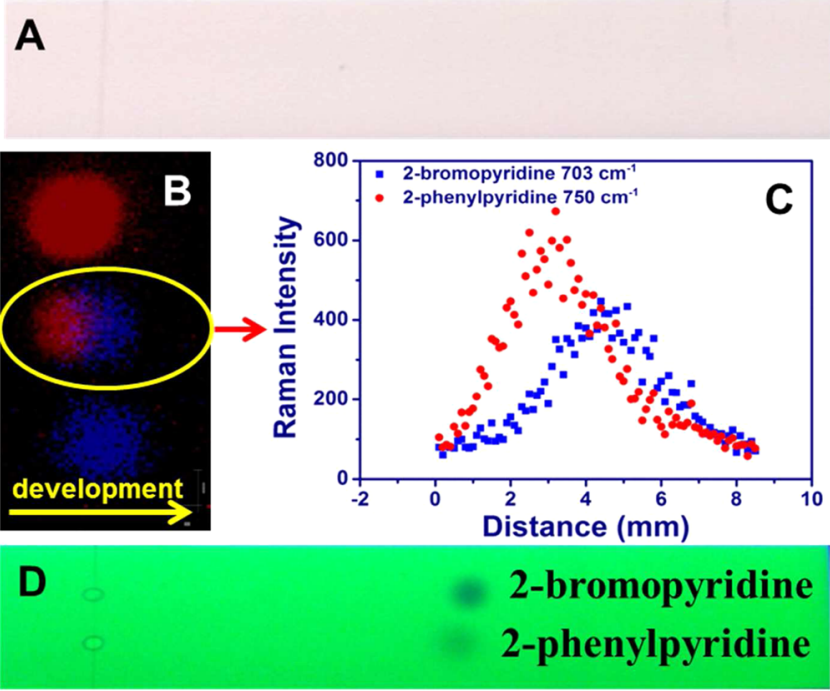

Figure 4. TLC separation and SERS detection of 2-bromopyridine and 2-phenylpyridine: (A) image of the G TLC plate after the development of 2-bromopyridine, 2-phenylpyridine, and the mixture; (B) SERS image of 2-bromopyridine, 2-phenylpyridine, and their mixture quantified by their characteristic Raman bands after the development with a G TLC plate; (C) Raman intensity distribution of 2-bromopyridine and 2-phenylpyridine along the line across the middle of mixture spot; and (D) fluorescence image of the $\mathrm{GF}_{254}$ TLC plate after the development of 2-bromopyridine and 2-phenylpyridine.

that the two compounds, represent by different colors, have similar developing distances and overlapped in the mixture spot (Figure 4B). This was confirmed by the distribution profiles of the Raman intensity against distance acquired from their characteristic Raman bands along a line across the middle of the mixture spot on the G TLC plate (Figure 4C), as well as by the two black spots with similar $R_{\mathrm{f}}$ values observed under the 254$\mathrm{nm}$ UV light when the two chemicals were developed on the same $\mathrm{GF}_{254}$ TLC plate (Figure 4D). Notably, the above results demonstrated that, for products and reactants that are invisible for TCL only, and even have similar $R_{\mathrm{f}}$ values and cannot be separated completely on the TLC plate, the reaction process can be estimated through the relative intensity of their characteristic SERS bands. Thus, the TLC-SERS method has great potential in monitoring the reaction process, especially for the reactions with chemicals that are hardly separated or invisible to the eye for TLC only.

Identification of Unknown Compounds. The above success of TLC-SERS in identification of analytes in reacting mixtures encouraged us to test its applicability in identification of unknown compounds, e.g., byproducts or new complexes formed by catalysts and substrates. Thus, TLC-SERS detection was performed continuously from the point of sample to the solvent front, to determine if byproducts formed in the Suzuki coupling reaction of phenylboronic acid and 2-bromopyridine. To our delight, a strong SERS signal, which cannot be assigned to the reactants or products (see Figure S6 in the Supporting Information), was found at the point of the sample (Figure 5 (left)), indicating that a byproduct did form. Considering that, under alkaline conditions, the self-coupling between the reactants may occur in the Suzuki coupling reaction, ${ }^{28,29}$ the unknown Raman signal might come from biphenyl or 2,2bipyridine. The high similarity between the unknown Raman signal and that of 2,2-bipyridine (Figure 5 (right)) suggests that the unknown compound is relevant to 2,2-bipyridine. There- 


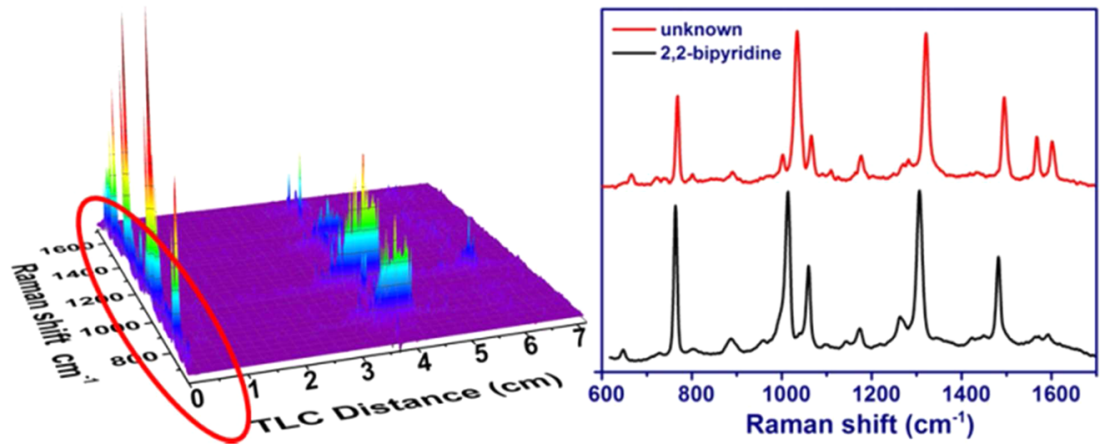

Figure 5. (Left) Full Raman mapping of the product mixture after the separation on the plate. (Right) Comparison of the Raman spectra of the unknown compound with 2,2-bipyridine.

fore, a series of experiments were conducted to identify the unknown compound.

Three pairwise combinations of the two reactants (phenylboronic acid, 2-bromopyridine) and catalyst (palladium acetate) were added into 3 three-necked bottles, respectively, to conduct the reaction under the same conditions. TLC-SERS detection results showed that the unknown SERS signal was detected only in the reaction mixture of 2-bromopyridine and palladium acetate, demonstrating that the unknown compound is related to the 2-bromopyridine. Because of the high similarity of the Raman signal between the unknown compound and 2,2bipyridine, further experiments were carried out to ascertain whether the unknown signal came from 2-bromopyridine or 2,2-bipyridine. For this purpose, 2-bromopyridine and 2,2bipyridine were mixed, respectively, with palladium acetate to conduct the reaction under the same conditions. TLC-SERS detection results showed that the unknown SERS signal in the reaction solution of 2,2-bipyridine with palladium acetate was much stronger than that of 2-bromopyridine with palladium acetate (see Figure S7A in the Supporting Information), indicating that the unknown compound was more relevant to 2,2-bipyridine and the self-coupling of 2-bromopyridine occurred during the reaction process.

To further test if the acetate in the catalyst contributed to the unknown compound, three kinds of catalysts-including palladium acetate, palladium nitrate, and palladium chloridewere compared. The quite similar TLC-SERS detection results (see Figure S7B in the Supporting Information) suggested that the acetate was not contained in the constitutional formula of the unknown compound.

We also tested if the buffer contributed to the unknown compound by conducting the reaction in potassium phosphate ( $\mathrm{pH}$ 13.4), sodium carbonate $(\mathrm{pH} 12.0)$, and water ( $\mathrm{pH}$ 6.7), respectively. Results of TLC-SERS detection (see Figure S7C in the Supporting Information) suggested that the production of unknown compound was enhanced in alkalescent buffers, but the components of the buffer were not contained in the constitutional formula of the unknown compound.

Based on the above TLC-SERS results, it can be concluded that, most likely, the unknown SERS signal stemmed from the complex of 2,2-bipyridine and palladium. However, the exact formula of the unknown product remains a topic for further study, because of the lack of standard SERS spectra and standards of the complexes of 2,2-bipyridine and palladium for validation.

\section{CONCLUSIONS}

By spraying gold nanoparticles (AuNPs) on a thin layer chromatography (TLC) plate with preseparated analytes to form surface-enhanced Raman scattering (SERS) substrates, we have developed a facile TLC-SERS method for on-site quantitative monitoring of chemical reactions. This method enables continuous detection in a line and a large area on TLC plate, as well as separation and detection on the same TLC plate to provide the concentration information on the analyte. Optimization of the AuNP size and concentration led to the evenly distributed and highly efficient SERS response on the surface of the plate, which enhanced the method sensitivity and precision. By continuous detecting along a line, this method provides full and accurate information about the process in specific chemical reaction, without missing substances that even are invisible to the naked eyes. Importantly, using the characteristic band of the Raman spectra, this proposed TLCSERS can also distinguish analytes that cannot be separated completely by TLC. Furthermore, through the full scan of a large area on the plate, new byproducts can be found and their primary structures can be identified by the Raman spectra. Thus, this facile TLC-SERS method can be exploited in monitoring the progresses of reaction, as well as environmental and biological process.

\section{ASSOCIATED CONTENT}

\section{S Supporting Information}

Experimental details and additional results are provided as Supporting Information. This material is available free of charge via the Internet at http://pubs.acs.org.

\section{AUTHOR INFORMATION}

\section{Corresponding Author}

*Tel./Fax: +86 10 62849192. E-mail address: jfliu@rcees.ac.cn. Notes

The authors declare no competing financial interest.

\section{ACKNOWLEDGMENTS}

This work is supported by the Chinese Academy of Sciences (No. YZ201147), the National Natural Science Foundation of China (Nos. 21025729, 21207124), and the Strategic Priority Research Program of the Chinese Academy of Sciences (No. XDB14020101).

\section{REFERENCES}

(1) Sherma, J. Anal. Chem. 2000, 72, 9R-25R.

(2) Sherma, J. Anal. Chem. 2010, 82, 4895-4910. 
(3) Stoll, M. S.; Hounsell, E. F.; Lawson, A. M.; Chai, W.; Feizi, T.

Eur. J. Biochem. 1990, 189, 499-507.

(4) Bonnett, R.; Czechowski, F.; Latos-Grazynski, L. Energy Fuels 1990, 4, 710-716.

(5) Nie, S. M.; Emery, S. R. Science 1997, 275, 1102-1106.

(6) Barber, T. E.; List, M. S.; Haas, J. W.; Wachter, E. A. Appl. Spectrosc. 1994, 48, 1423-1427.

(7) Zhang, Z. M.; Liu, R.; Xu, D. M.; Liu, J. F. Acta Chim. Sinica 2012, 70, 1686-1689.

(8) Li, J. F.; Huang, Y. F.; Ding, Y.; Yang, Z. L.; Li, S. B.; Zhou, X. S.; Fan, F. R.; Zhang, W.; Zhou, Z. Y.; Wu, D. Y.; Ren, B.; Wang, Z. L.; Tian, Z. Q. Nature 2010, 464, 392-395.

(9) Brosseau, C. L.; Gambardella, A.; Casadio, F.; Grzywacz, C. M.; Wouters, J.; Van Duyne, R. P. Anal. Chem. 2009, 81, 3056-3062.

(10) Hezel, U. B.; Zeiss, C. In Journal of Chromatography Library, Zlatkis, A., Kaiser, R. E., Eds.; Elsevier: Amsterdam, 1977; Vol. 9, Chapter 8.

(11) Caudin, J. P.; Beljebbar, A.; Sockalingum, G. D.; Angiboust, J. F.; Manfait, M. Spectrochim. Acta, Part A 1995, 51, 1977-1983.

(12) Matejka, P.; Stavek, J.; Volka, K.; Schrader, B. Appl. Spectrosc. 1996, 50, 409-414.

(13) Zhu, Q. X.; Cao, Y. B.; Cao, Y. Y.; Chai, Y. F.; Lu, F. Anal. Bioanal. Chem. 2014, 406, 1877-1884.

(14) Yao, C. P.; Cheng, F. S.; Wang, C.; Wang, Y. H.; Guo, X. W.; Gong, Z. J.; Fan, M. K.; Zhang, Z. Y. Anal. Methods 2013, 5, 55605564.

(15) Wang, Y. Q.; Yan, B.; Chen, L. X. Chem. Rev. 2013, 113, 13911428.

(16) Pozzi, F.; Shibayama, N.; Leona, M.; Lombardi, J. R. J. Raman Spectrosc. 2013, 44, 102-107.

(17) Lucotti, A.; Tommasini, M.; Casella, M.; Morganti, A.; Gramatica, F.; Zerbi, G. Vib. Spectrosc. 2012, 62, 286-291.

(18) Huang, R. F.; Han, S. Y.; Li, X. Anal. Bioanal. Chem. 2013, 405, 6815-6822.

(19) Li, D. W.; Qu, L. L.; Zhai, W. L.; Xue, J. Q.; Fossey, J. S.; Long, Y. T. Environ. Sci. Technol. 2011, 45, 4046-4052.

(20) Freye, C. E.; Crane, N. A.; Kirchner, T. B.; Sepaniak, M. J. Anal. Chem. 2013, 85, 3991-3998.

(21) Frens, G. Nature 1973, 241, 20-22.

(22) Zawada, K.; Bukowska, J.; Calvo, M.; Jackowska, K. Electrochim. Acta 2001, 46, 2671-2680.

(23) Zawada, K.; Bukowska, J. Surf. Sci. 2002, 507, 34-39.

(24) Piergies, N.; Proniewicz, E.; Ozaki, Y.; Kim, Y.; Proniewicz, L. M. J. Phys. Chem. A 2013, 117, 5693-5705.

(25) Leff, D. V.; Ohara, P. C.; Heath, J. R.; Gelbart, W. M. J. Phys. Chem. 1995, 99, 7036-7041.

(26) Liu, J. F.; Liu, R.; Yin, Y. G.; Jiang, G. B. Chem. Commun. 2009, 12, 1514-1516.

(27) Boopalachandran, P.; Sheu, H.-L.; Laane, J. J. Mol. Struct. 2012, 1023, 61-67.

(28) Arcadi, A.; Cerichelli, G.; Chiarini, M.; Correa, M.; Zorzan, D. Eur. J. Org. Chem. 2003, 2003, 4080-4086.

(29) Duan, X. F.; Li, X. H.; Li, F. Y.; Huang, C. H. Synth. Commun. 2004, 34, 3227-3233. 